\title{
An immune-related long non-coding RNA signature predicts prognosis in glioblastoma patients
}

Xiangdong $\mathrm{Lu}^{1,2,3}$, Siquan $\mathrm{Zhu}^{3}$, Shouqing Zhang ${ }^{3}$, Feng $\mathrm{Si}^{3}$, Yunfeng $\mathrm{Ma}^{3}$, Fei Chen ${ }^{3}$, Peng Qiu ${ }^{4}$

\begin{abstract}
${ }^{1}$ Nanchang University, Nanchang 330031, Jiangxi, China
2Department of Neurosurgery, Jiangxi provincial People's Hospital The First Affiliated Hospital of Nanchang Medical College, Nanchang 330006, Jiangxi, China

${ }^{3}$ Department of Neurosurgery, Department of Neurosurgery, People's Hospital Affiliated to Shandong First Medical University, Jinan271100, Shandong, China ${ }^{4}$ Department of Neurosurgery, Shandong Provincial Hospital Affiliated to Shandong First Medical University, Jinan, Shandong Provincial, China
\end{abstract}

Submitted: 24 April 2021; Accepted: 21 July 2021

Online publication: 27 July 2021

Arch Med Sci

DOI: https://doi.org/10.5114/aoms/140397

Copyright @ 2021 Termedia \& Banach

\section{Abstract}

Introduction: This study aimed to explore the prognostic value of immune-related long non-coding RNAs (IncRNAs) in glioblastoma (GBM).

Material and methods: Expression and clinical data were acquired, including the GSE111260 dataset: $67 \mathrm{GBM}$ and 3 normal brain samples; the GSE103227 dataset: 5 GBM and 5 normal brain samples; and TCGA data: 187 GBM samples. Immune-related genes were retrieved from the ImmPort database. Immune-related differentially expressed genes (DEGs) and IncRNAs were screened. Prognostic IncRNAs were then screened to establish a prognostic risk score model. Survival analysis and differential expression analysis were performed in high- vs. low-risk groups, followed by the protein-protein interaction network and the IncRNA-mRNA co-expression network.

Results: A total of 251 immune-related DEGs were screened. After correlation analysis, 387 immune-related IncRNAs that co-expressed with 140 immune-related DEGs were screened. Univariate analysis identified 18 IncRNAs that were significantly associated with prognosis. The prognostic risk score was able to stratify GBM patients into high- and low-risk groups, and patients with high risk scores displayed worse outcomes than those with low risk scores in both the training set and the validation set. A total of 272 genes had abnormal expression between high- and low-risk groups. Of these, 22 genes were immune-related, such as SNAP25, SNAP91, $S N C B$, and RAB3A. These genes were mainly enriched in synaptic vesicle cycle/exocytosis and insulin secretion. The co-expression network contained 22 genes and 11 IncRNAs, and IncRNA LINC01574 co-expressed with the great number of mRNAs.

Conclusions: We identified 18 immune-related prognostic IncRNAs, and the established IncRNA-based prognostic risk model could stratify GBM patients into different risk levels.

Key words: glioblastoma, long non-coding RNA, immune, prognosis.

\section{Introduction}

Glioma is the most common malignant tumor of the central nervous system (CNS). As the most malignant pathological type of glioma, glioblastoma (GBM) has strong aggressiveness and a high recurrence rate, accounting for approximately $52 \%$ of gliomas [1]. At present, the standard treatment for GBM is still surgical resection supplemented by ra-

\author{
Corresponding author: \\ Peng Qiu \\ Department of Neurosurgery \\ Shandong Provincial Hospital \\ Shandong First Medical \\ University \\ No.324, Jingwu Road, \\ Jinan 250000 \\ Shandong Provincial, China \\ Phone/fax: \\ +86-053168773225 \\ E-mail: Qpk1007@163.com
}


diotherapy and chemotherapy [2]. However, it is difficult to completely excise the tumor due to the invasive growth of GBM tumor cells and the restriction of the tumor site. Moreover, chemoradiotherapy cannot specifically identify tumor cells and may result in drug resistance. Therefore, the prognosis of GBM is still poor with a overall median survival of 15 months and a 5-year survival rate less than $10 \%$ [3]. The CNS has long been known to be an immunologically privileged region [4]. However, in recent years, a large number of studies have shown that the immune system in the brain is in a high level of activation and can interact with brain tumors [5]. Therefore, it is very important to investigate the immune landscape for the treatment of GBM.

Immune cells represent the main population of non-neoplastic cells in the tumor microenvironment of GBM, and different GBM subtypes show differences in their microenvironment [6]. Identification of molecular markers has been demonstrated to effectively distinguish tumor subtypes, degree of malignancy, and response to chemoradiotherapy [7]. For example, Zhou et al. found that high expression of ribonucleotide reductase subunit M2 (RRM2) was associated with poor survival of patients with GBM, suggesting that RRM 2 could be a prognostic biomarker in GBM [8]. Han et al. suggested that the interaction of miR-431-5perythrocyte membrane protein band 4.1 (EPB41L1) is involved in GBM progression, implying the potential of miR-431-5p and EPB41L1 in predicting tumor progression [9]. Long non-coding RNAs (IncRNAs) act as major regulators of gene expression, and dysregulation of IncRNAs is involved in a variety of biological processes in many cancers [10, 11]. Aberrant expression of IncRNAs participates in brain glioma development and may be a critical regulator of tumorigenesis [12]. Mazor et al. reported that high expression of IncRNA TP73 antisense RNA 1 (TP73-AS1) predicted aggressiveness and poor prognosis of GBM and might contribute to temozolomide resistance in GBM cancer stem cells [13]. Lu et al. observed that over-expression of IncRNA small nucleolar RNA host gene 12 (SNHG12) promoted the cells to acquire temozolomide resistance, which could predict poor survival for GBM patients [14]. Despite these observations, the immune-related IncRNAs that have prognostic value remain largely unknown.

In this study, we identified immune-related IncRNAs associated with the prognosis of GBM, constructed an immune-related IncRNA signature-based prognostic risk score model, and explored the underlying differences in molecular expression, biological function and regulatory mechanism between high- and low-risk groups. Overall, we hypothesized that the identified im- mune-related IncRNAs played pivotal roles in GBM progression, and the identified prognostic signature of 18 immune-related IncRNAs could be a novel prognostic biomarker and therapeutic target for GBM, contributing to improved prognosis prediction and treatment of this disease.

\section{Material and methods}

\section{Dataset acquisition and preprocessing}

GSE111260 and GSE103227 datasets were downloaded from the GEO database [15]. GSE111260 contained 70 human samples, including 67 glioma samples and 3 normal brain samples. The data were generated on the GPL5175 [HuEx-1_0-st] Affymetrix Human Exon 1.0 ST Array platform. GSE103227 contained 5 GBM samples and 5 normal brain samples, and the platform was GPL16956 Agilent-045997 Arraystar human IncRNA microarray V3. The data were preprocessed in standardized fashion utilizing the robust multi-array average (RMA) method [16] in the Limma package [17] in R3.4.3. The probes were annotated on the basis of the annotation files of the platform. The probes matching no gene symbol were removed, and the mean value was selected as the final expression value of the gene when multiple probes matched to one gene symbol. In addition, the FPKM RNA-seq data and clinical phenotype data of 167 GBM samples were obtained from The Cancer Genome Atlas (TCGA) database. The Ensemble gene was converted into a gene symbol utilizing the annotation file (hg38, gencode.V22.) of the GENCODE database [18]. The mean value was selected as the final expression value of the gene when multiple Ensemble genes matched to one gene symbol. Then mRNAs and IncRNAs were obtained based on the gene symbol types, of which mRNAs and IncRNAs without expression in more than $80 \%$ of samples were removed. These three datasets (GSE111260, GSE103227 and TCGA-GBM datasets) were integrated and analyzed in this study, and the analysis procedure is shown in Supplementary Figure S1.

\section{Screening of differentially expressed genes (DEGs) and immune-related DEGs}

Differential expression analysis in GBM vs. normal brain samples was carried out utilizing the Limma package in R 3.4.3, and genes with |logFC| $>1$ and $p$ value $<0.05$ were considered as DEGs. The common up-regulated and down-regulated DEGs from the two databases (GSE111260 and GSE103227) were screened and used in the following analysis. The immune-related genes in The Immunology Database and Analysis Portal (ImmPort) database were downloaded from InnateDB [19] (https://www.innatedb.ca/index.jsp). 
By Venn analysis, the overlapped genes between immune-related genes and DEGs were screened as immune-related DEGs.

\section{Screening of immune-related IncRNAs}

The expression data of immune-related DEGs in the TCGA-GBM dataset were retrieved. Correlation analysis between immune-related DEGs with all annotated IncRNAs was then conducted utilizing cor.test in R 3.4.3. Immune-related IncRNAs were screened by |Pearson correlation coefficient| $>0.6$ and $p$ value $<0.001$.

\section{Construction of IncRNA-based prognostic risk model}

Among the 167 GBM samples, seven samples were removed due to their survival time being less than one month, and the remaining samples were randomly divided into the training set $(n=$ $80)$ and the validation set $(n=80)$ with a ratio of $5: 5$. Univariate Cox regression analysis was performed to screen the immune-related IncRNA that was significantly correlated with prognosis. Then, the IncRNAs with log-rank $p<0.05$ in univariate analysis were further included in multivariate Cox regression analysis.

Then the prognostic risk model was constructed according to the formula: Risk score $=\sum$ Coef IncRNAs $\times$ Exp IncRNAs, where Coef represents the correlation coefficient in multivariate Cox regression analysis, and Exp represents the expression value of the IncRNAs in the TCGA dataset. Patients were divided into high- and low-risk groups based on the risk score calculated by the prognostic risk model, and Kaplan-Meier survival curves and the log-rank test were utilized to conduct survival analysis to confirm the prognostic value of the risk model in the training set, validation set and total set (training set + validation set).

\section{DEGs in high-risk and low-risk group}

Based on the risk score calculated by the prognostic risk model, samples in the TCGA set were divided into high- and low-risk groups. DEGs in high- vs. low-risk were screened utilizing the Limma package with the cut-off value of |log2FC| $>0.585$ and $p<0.05$. The overlapped genes between DEGs and immune-related genes from the ImmPort database were screened as key immune DEGs, and functional enrichment analysis was performed for these overlapped genes. The clusterProfiler tool [20] was utilized to conduct functional enrichment analysis, including Gene Ontology (GO) annotation terms and KEGG pathways. The significant enriched results were screened by threshold of count $\geq 2$ and $p<0.05$. Additionally, the protein-protein interactions (PPIs) among those genes were predicted utilizing the STRING database [21] with a PPI score of 0.4. The PPI network was visualized by Cytoscape [22].

\section{Construction of IncRNA-mRNA co-expression network}

Correlation analysis between key immune DEGs and IncRNA was conducted, and IncRNA-mRNA pairs with |Pearson correlation coefficient $\mid>0.3$ and $p$ value $<0.05$ were screened to construct the IncRNA-mRNA co-expression network. To further investigate the function of these IncRNAs, clusterProfiler was utilized to explore the involved KEGG pathways of mRNAs in the IncRNA-mRNA network. The significant enriched pathways were screened with $p<0.05$.

\section{Results}

\section{Screening of DEGs and immune-related DEGs}

A total of 5569 DEGs between GBM and normal brain samples were screened from the GSE103227 dataset, including 2886 up- and 2683 down-regulated genes (Figure 1 A). Moreover, 1429 DEGs (including 558 up- and 871 down-regulated) were screened from the GSE111260 dataset (Figure 1 B). Venn analysis showed that there were 819 overlapped DEGs in the two datasets, including 257 up- and 562 down-regulated overlapped DEGs (Figure $1 \mathrm{C}$ ).

In order to screen the immune-related genes from the DEGs, 4677 immune-related genes were retrieved from the ImmPort database. A total of 215 overlapped genes between immune-related genes and DEGs were identified as immune-related DEGs (Figure $1 \mathrm{D}$ ).

\section{Identification of immune-related IncRNAs by correlation analysis}

A total of 5934 IncRNAs and 16863 mRNAs were annotated from the TCGA-GBM dataset. By correlation analysis of 4677 immune-related genes and 5934 IncRNAs, 1902 IncRNA-mRNA co-expression pairs were obtained, involving 387 IncRNAs and 140 immune-related DEGs. These 387 IncRNAs were considered as immune-related IncRNAs.

\section{Construction of IncRNA-based prognostic risk model}

The results of univariate Cox regression analysis showed that 18 immune-related IncRNAs were significantly associated with prognosis (Table I), which were further included in multivariate Cox regression analysis to calculate the $\beta$ coefficient (Coef in formula) (Table II). 
A Cutoff for logFC is 1

The number of up-regulated genes is 2886

The number of down-regulated genes is 2683

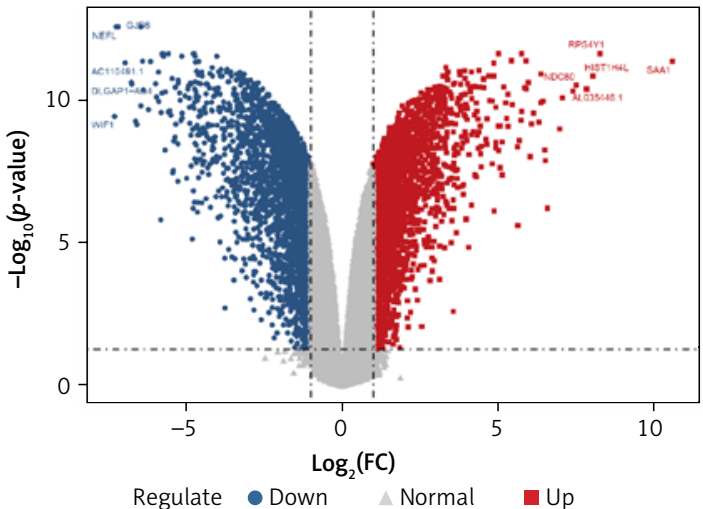

C

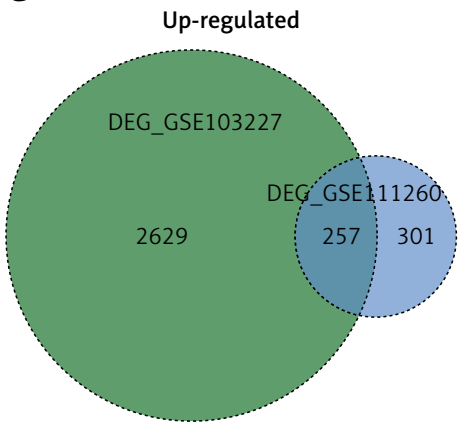

B Cutoff for logFC is 1

The number of up-regulated genes is 558

The number of down-regulated genes is 871

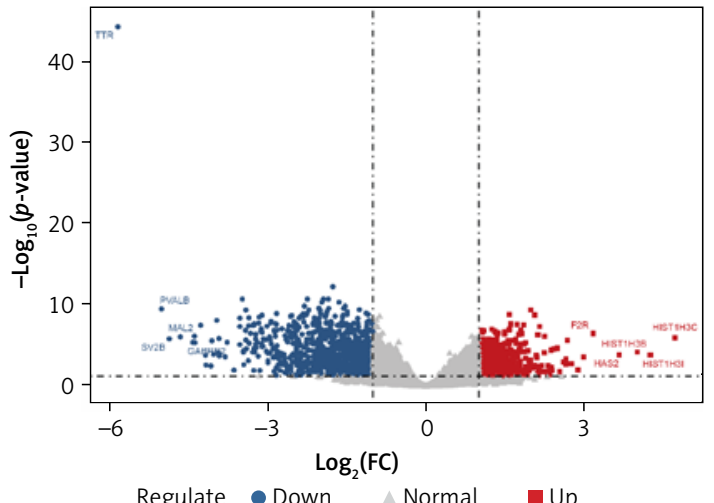

D
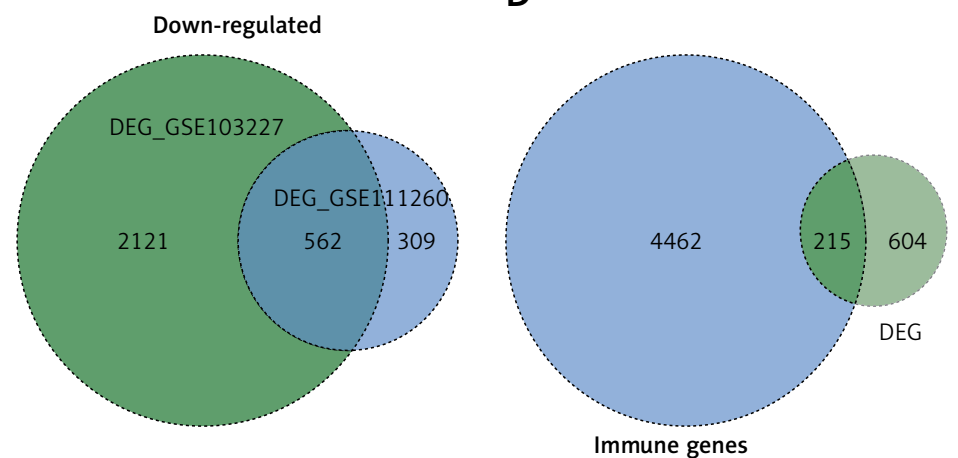

Figure 1. Screening of differentially expressed genes (DEGs) between glioblastoma (GBM) and normal brain samples and immune-related DEG. Volcano plots of DEGs in GSE103227 dataset (A) and GSE111260 dataset (B); Venn plot shows the overlapped DEGs in GSE103227 and GSE111260 datasets (C); Venn plot shows the overlapped genes between immune-related genes from ImmPort database and overlapped DEGs in the two datasets (D)

Table I. The 18 immune-related prognosis IncRNAs screened by univariate Cox regression analysis

\begin{tabular}{|lcccc|}
\hline Symbol & HR & Lower.95 & Upper.95 & $P$-value \\
\hline LINC01574 & 3.054672 & 1.364737 & 6.837233 & 0.006599 \\
\hline AC011899.9 & 1.994071 & 1.200441 & 3.312384 & 0.007687 \\
\hline SOX21-AS1 & 0.587738 & 0.391603 & 0.882107 & 0.010303 \\
\hline RP11-344B5.2 & 1.554721 & 1.076846 & 2.244665 & 0.018519 \\
\hline CTD-2540B15.6 & 1.675293 & 1.088034 & 2.579523 & 0.019124 \\
\hline RP11-455F5.5 & 3.817241 & 1.201353 & 12.1291 & 0.023149 \\
\hline AC093627.10 & 2.129741 & 1.1052 & 4.104053 & 0.023894 \\
\hline AF064858.6 & 34383.28 & 3.95651 & $2.99 E+08$ & 0.023997 \\
\hline RP11-452C13.1 & 2.393516 & 1.118737 & 5.120882 & 0.024506 \\
\hline LINC00323 & 2.367093 & 1.114584 & 5.027101 & 0.024945 \\
\hline RP5-899E9.1 & 2.252868 & 1.087417 & 4.667404 & 0.028855 \\
\hline RP6-159A1.4 & 1.568564 & 1.046889 & 2.350194 & 0.029103 \\
\hline LINC01480 & 1.706491 & 1.04476 & 2.787349 & 0.032771 \\
\hline LLNLR-470E3.1 & 1.395112 & 1.018068 & 1.911796 & 0.038325 \\
\hline RP11-1398P2.1 & 1.901376 & 1.034757 & 3.493797 & 0.03845 \\
\hline L3MBTL4-AS1 & 2.22636 & 1.04099 & 4.761505 & 0.039062 \\
\hline ZBED5-AS1 & 1.528917 & 1.016631 & 2.299348 & 0.041432 \\
\hline RP11-350G8.5 & 2.90388 & 1.036241 & 8.13761 & 0.042593 \\
\hline
\end{tabular}


Prognostic risk score models were established in the training set, validation set and total set (training set + validation set). Then, the risk score was calculated for each sample, and patients were divided into high- and low-risk groups based on the median risk score. Kaplan-Meier survival curves showed that patients with high risk scores had a shorter survival than those with low risk scores in the training set $(p<0.0001)$, validation set $(p=0.024)$ and total set $(p<0.0001)$ (Figure 2). These data suggested that the risk score model could stratify GBM patients with different prognoses.

\section{Screening of DEGs in high- vs. low-risk groups}

All the TCGA-GBM samples were divided into high- and low-risk groups based on the median risk score. A total of 272 DEGs in high- vs. low risk groups were obtained, including 58 up- and 214 down-regulated genes (Figure $3 \mathrm{~A}$ ). Among the 272 DEGs, there were 22 immune-related DEGs after merging with the 4677 immune-related genes. To explore the interactions among proteins encoded by these 22 immune-related DEGs, the PPI network was constructed (Figure $3 \mathrm{~B}$ ). The PPI network contained 18 nodes and 53 interactions. SNAP25 (synaptosome associated protein 25 , degree $=12$ ), SYT1 (synaptotagmin 1, degree $=10$ ), SNCB (synuclein beta, degree $=10$ ), SNAP91 (Ras-related protein rab-3A, degree $=9$ ) and RAB3A (degree $=8$ ) were considered as hub nodes in this PPI network with a higher degree.

Functional enrichment analysis of immunerelated DEGs in high- vs. low-risk groups

We further investigated the involved GO annotation terms and KEGG pathways of the $22 \mathrm{im}$ mune-related DEGs. A total of 174 GO-biological processes were enriched, such as the synaptic vesicle cycle, synaptic vesicle exocytosis, neurotransmitter secretion and transport (Figure $4 \mathrm{~A}$ ). A total of 53 GO-cellular component annotations were enriched, such as glutamatergic synapse, synaptic vesicle and distal axon. In terms of GO-molecular function, SNARE binding, syntaxin-1 binding and others such as protein C-terminus binding were enriched (Figure $4 \mathrm{C}$ ). Additionally, two KEGG pathways, the synaptic vesicle cycle and insulin secretion, were significantly enriched (Figure 4 D).

\section{Construction of IncRNA-mRNA co-expression network}

Correlation analysis of the expression levels between 18 IncRNAs for prognosis risk model construction and the 22 immune-related DEGs was performed. As a result, 55 IncRNA-mRNA co-expression pairs were obtained, involving 22
Table II. The $\beta$ coefficient calculated by multivariate Cox regression analysis

\begin{tabular}{|lc|}
\hline LnCRNA & $\beta$ \\
\hline CTD-2540B15.6 & 0.38652 \\
\hline LLNLR-470E3.1 & 0.170048 \\
\hline RP11-1398P2.1 & -0.14419 \\
\hline RP11-344B5.2 & 0.355975 \\
\hline RP6-159A1.4 & -0.00388 \\
\hline L3MBTL4-AS1 & -0.44045 \\
\hline LINC01480 & 0.202784 \\
\hline RP11-350G8.5 & -1.15437 \\
\hline AC011899.9 & 1.418474 \\
\hline AC093627.10 & -0.91811 \\
\hline RP5-899E9.1 & -0.62939 \\
\hline LINC01574 & 1.373109 \\
\hline RP11-452C13.1 & 0.121207 \\
\hline LINC00323 & 0.344764 \\
\hline SOX21-AS1 & -0.82296 \\
\hline ZBED5-AS1 & 0.577906 \\
\hline AF064858.6 & 3.288317 \\
\hline RP11-455F5.5 & -1.16885 \\
\hline
\end{tabular}

mRNAs and 11 IncRNAs (Figure 5). Among 11 IncRNAs, long intergenic non-protein coding RNA 1574 (LINC01574) was co-expressed with the greatest number of immune-related DEGs, such as SNAP25, SYT1, SNCB, and SNAP91. Furthermore, these IncRNAs were involved in different $\mathrm{GO}$ biological processes and KEGG pathways. For example, LINC01574 was associated with GO functions associated with the synaptic vesicle cycle, vesicle-mediated transport in synapse, glutamate secretion, etc. RP11-1398P2.1 was implicated in GO functions associated with positive regulation of cyclin-dependent protein kinase activity, and cerebral cortex/telencephalon/forebrain cell migration. LINC01574 was involved in key pathways, such as the synaptic vesicle cycle and insulin secretion, and RP11-455F5.5 was enriched in insulin secretion and neuroactive ligand-receptor interaction pathways (Figure 6).

\section{Discussion}

GBM is the deadliest brain malignancy with poor outcomes due to the limited effect of existing treatments, including surgical and chemoradiotherapy [23]. Identification of key molecular markers affecting the prognosis of GBM helps to optimize the treatment and improve the clinical outcome. In the present study, 18 immune-related IncRNAs were identified to have a significant impact on the prognosis of patients. The prognostic risk score model integrating these 18 immune-related IncRNAs was constructed, which could strat- 
A

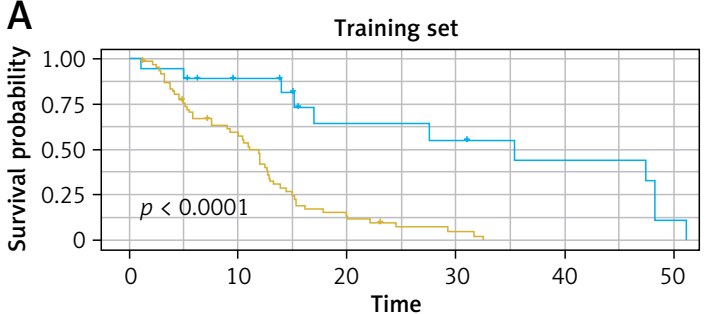

Number at risk
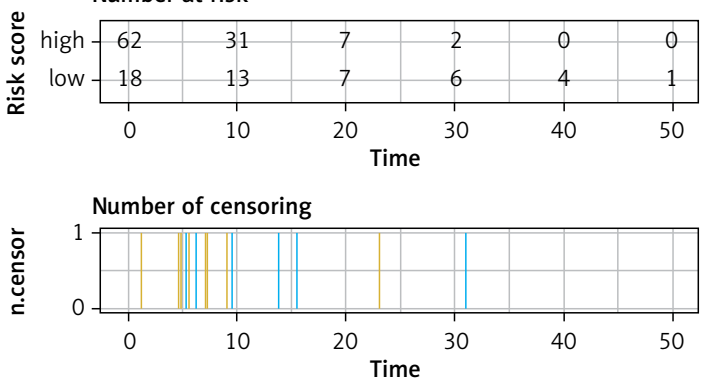

Risk score $\quad+$ Risk score high + Risk score low

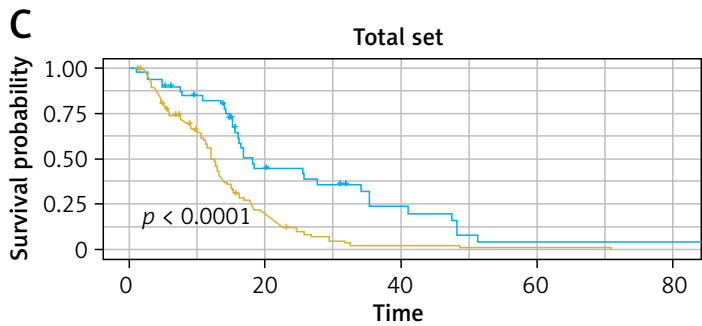

Number at risk
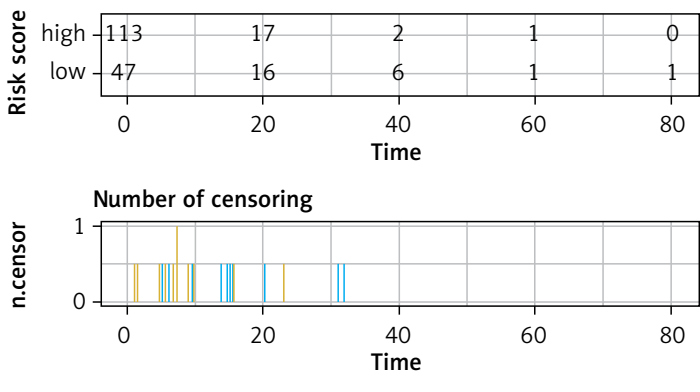

Risk score $\quad-$ Risk score high $\quad+$ Risk score low

ify GBM patients into groups with different risks and predict the prognosis of patients. Furthermore, IncRNA LINC01574 was co-expressed with more immune-related DEGs between high- and low-risk groups, such as SNAP25, SNAP91, SNCB and $R A B 3 A$. Additionally, the IncRNAs in the co-expression network were enriched in different biological functions and pathways.

In recent years, immunotherapy has successfully altered the landscape for the treatment of many types of cancers, including GBM [24]. Nevertheless, the initial immunotherapy trials show dismal results in GBM because of the complexities within intratumoral molecular heterogeneity [25]. Screening of key immune-related molecular markers is essential to design personalized immunotherapy for GBM. Increasing studies have suggested that
B
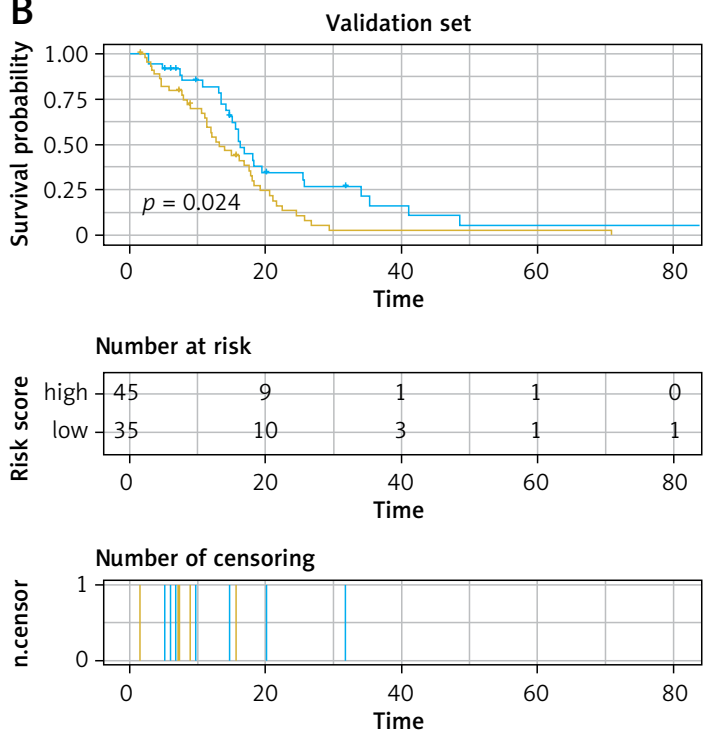

Risk score $\quad+$ Risk score high $\quad+$ Risk score low

Figure 2. Kaplan-Meier survival curves show the survival status of patients with high and low risk scores in training set (A), validation set (B) and total set (C)

immune-related IncRNAs could indicate prognosis of glioma patients [26, 27]. For example, Wang et al. reported that their identified 9-immune-related InCRNA signature could have a predictive role for anaplastic gliomas patients who had different OS and progression-free survival, and this signature was independent from other clinical factors [28]. Zhou et al. found that a 6-IncRNA signature could stratify GBM patients into high/low-risk groups with obvious diverse survival, and this signature was implicated in tumorigenesis by taking part in immune-related pathways [29]. Wang et al. established that a 5-immune-related IncRNA signature could stratify glioma patients into diverse tumor immune subtypes, and their expression showed positive correlations with immune indicators, such as programmed cell death-1 [30]. The iden- 
A Cutoff for logFC is 0.585

The number of up-regulated genes is 58

The number of down-regulated genes is 214

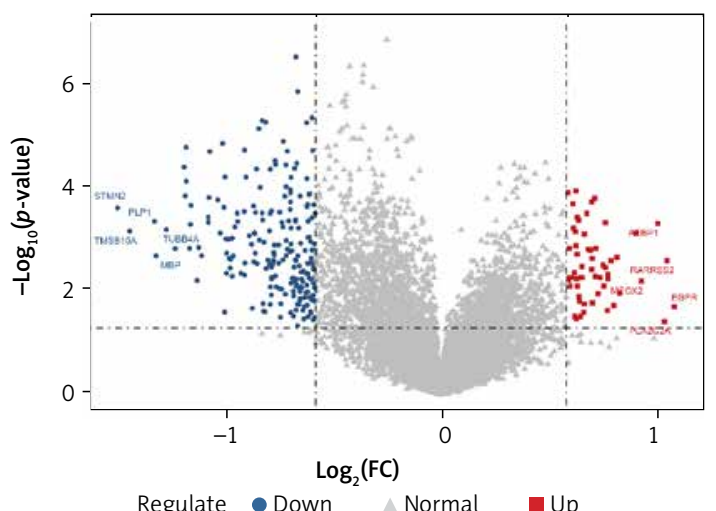

B

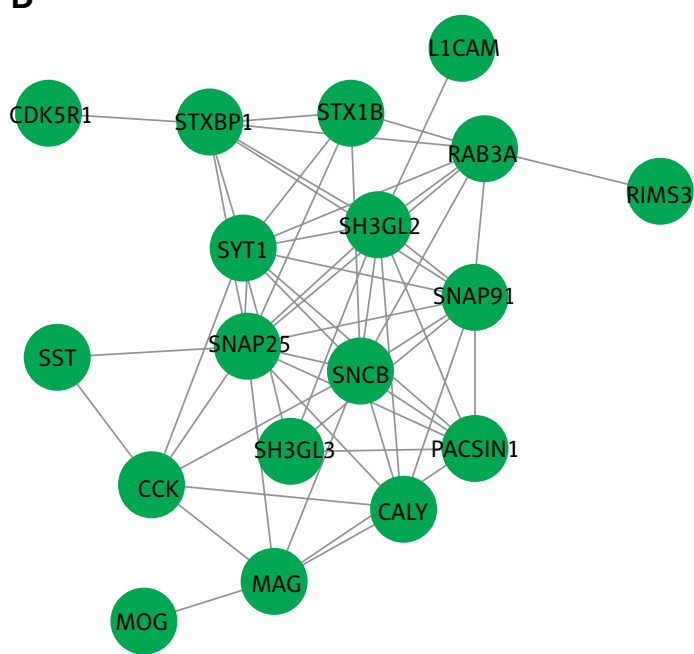

Figure 3. DEGs in high- vs. low-risk groups. Volcano plots of DEGs between high-risk and low-risk groups in TCGA-GBM dataset (A). Protein-protein interaction (PPI) network of immune-related DEGs between high-risk and low-risk groups (B). Green nodes represent down-regulated immune-related DEGs

A

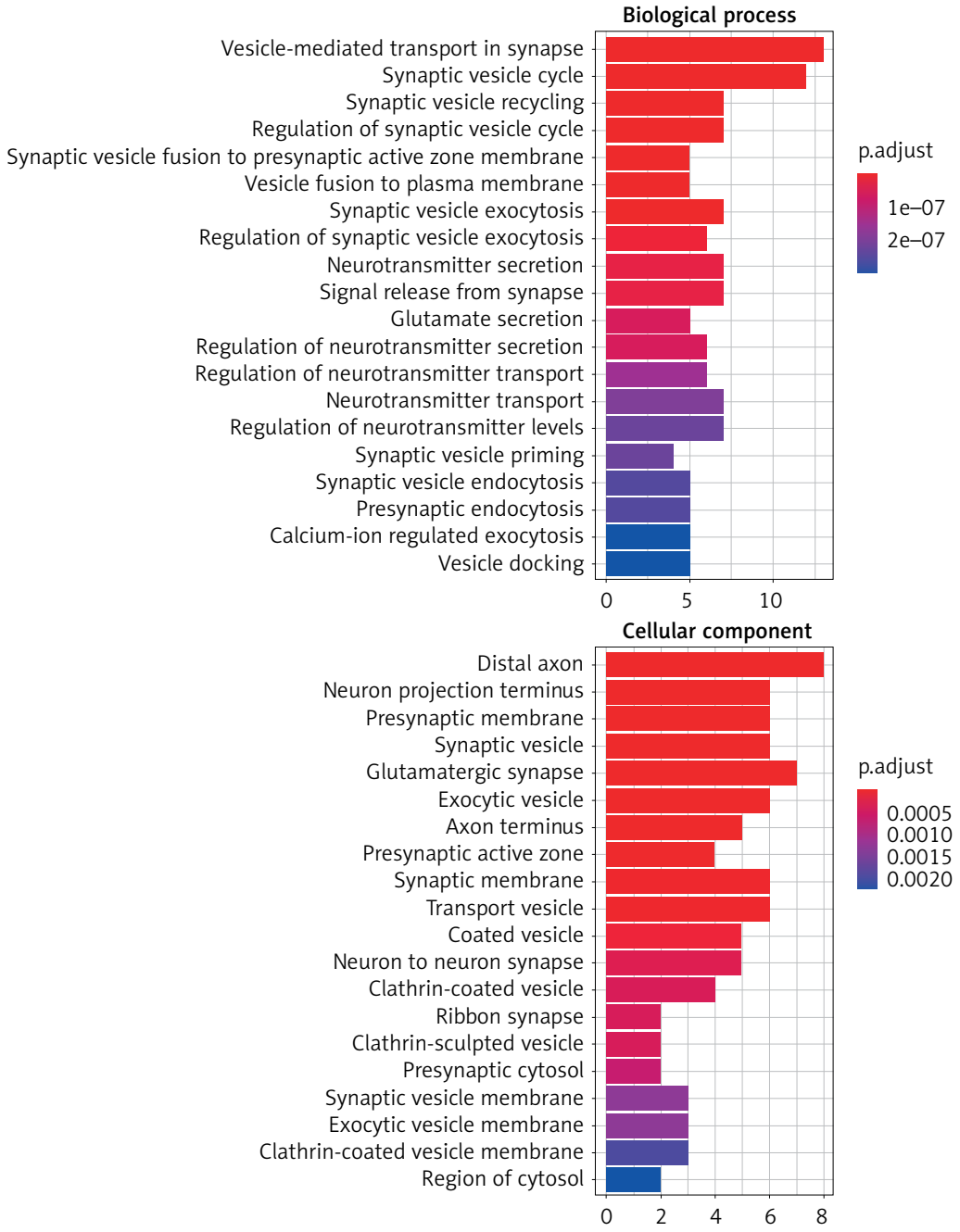

Figure 4. Functional enrichment analysis of immune-related DEGs in high- vs. low-risk groups. Histogram shows the top 20 enriched gene ontology (GO) biological processes (A), cellular component (B) 
C

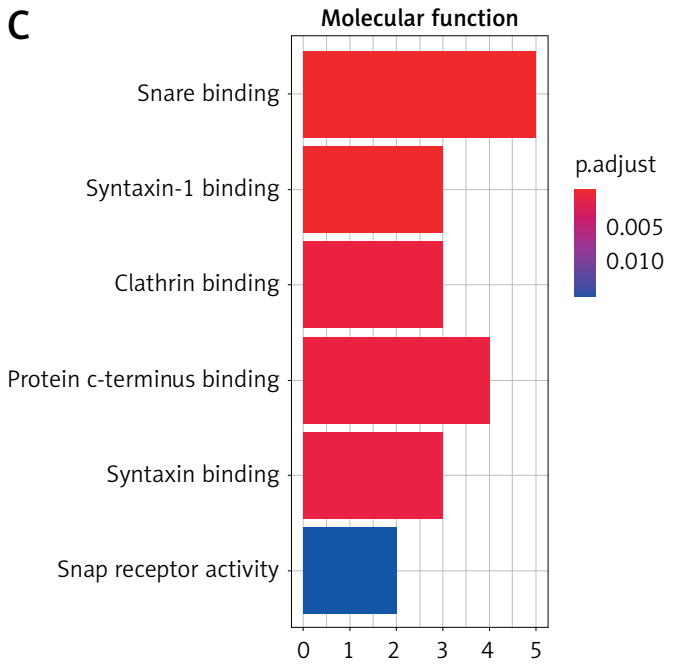

D

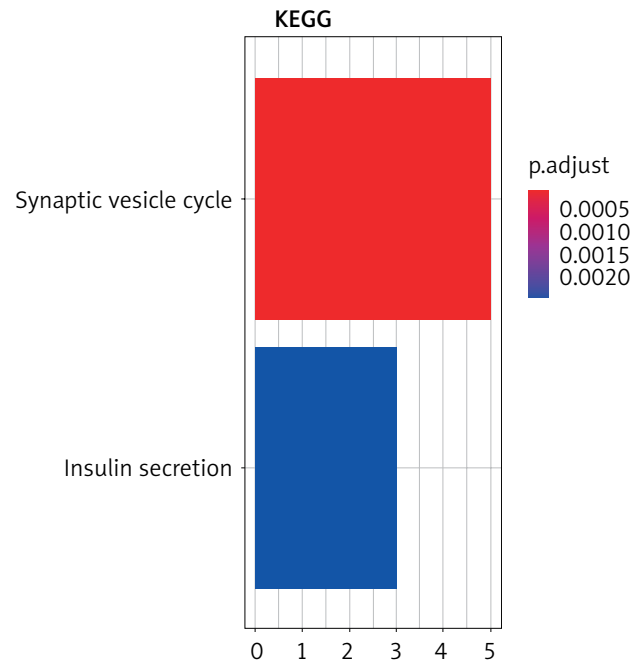

Figure 4. Cont. Molecular function (C), and KEGG pathways (D)

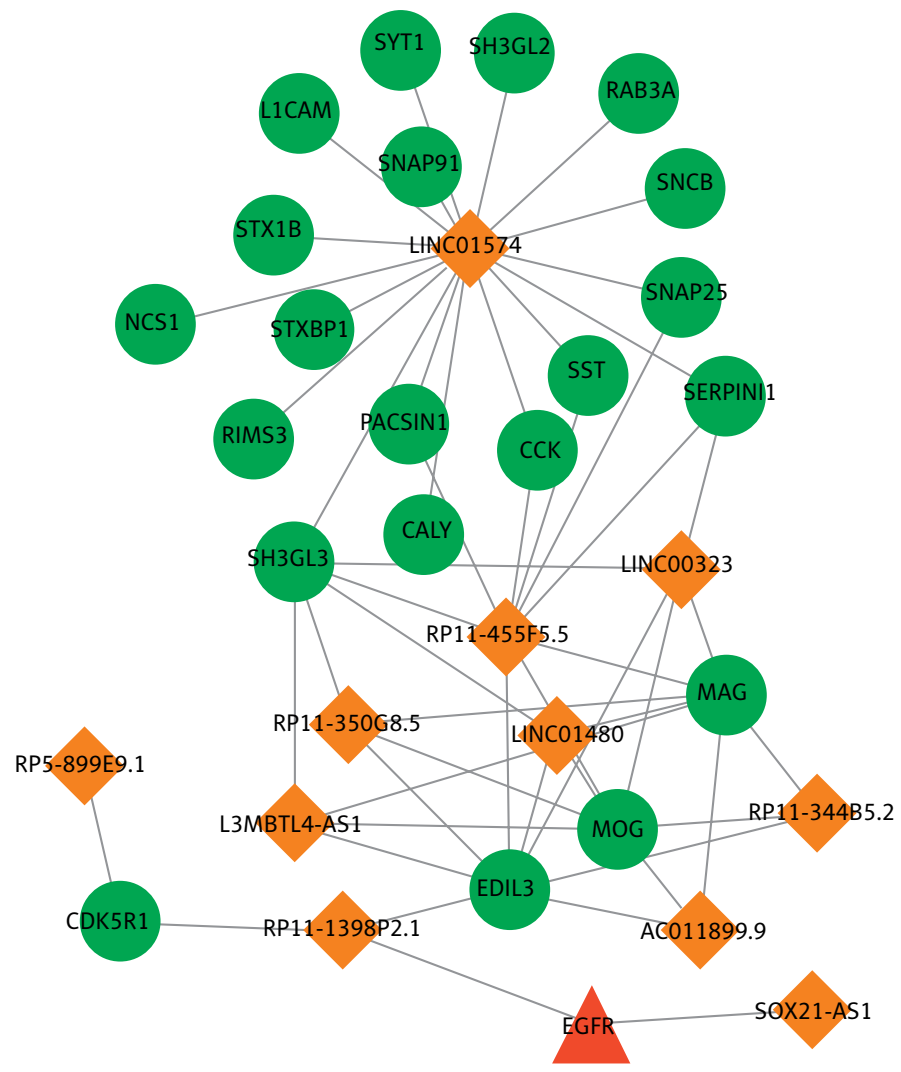

Figure 5. The IncRNA-mRNA co-expression network. Green circles and red triangles represent down-regulated and up-regulated immune-related DEGs, respectively; orange rhombus represent IncRNAs

tified prognostic signature of 18 immune-related IncRNAs in this study could stratify GBM patients into groups with different risks and predict the prognosis of patients. Given the low 5 -year survival rate (less than 10\%) [3], we believe that the constructed prognostic risk score model based on an 18-immune-related IncRNA signature could guide doctors to judge the disease severity and evaluate the prognosis, and contribute to the de- velopment of more reasonable and effective personalized treatment plans, so as to maximize the benefits of GBM patients.

Furthermore, IncRNA LINC01574 was co-expressed with more immune-related DEGs that were involved in the synaptic vesicle cycle, such as SNAP25, SNAP91, SNCB, and RAB3A. The communication among glioma tumor cells and non-glioma brain cells has been proved to be 
A

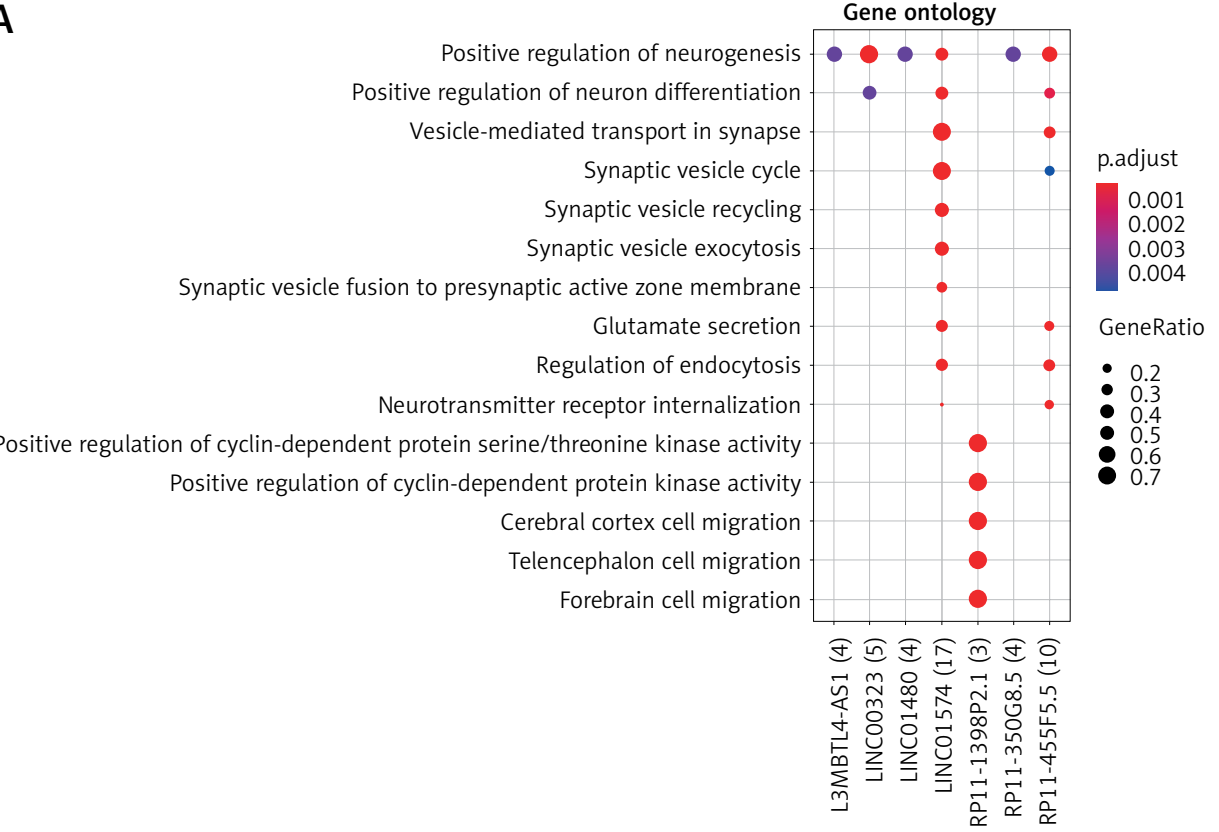

B

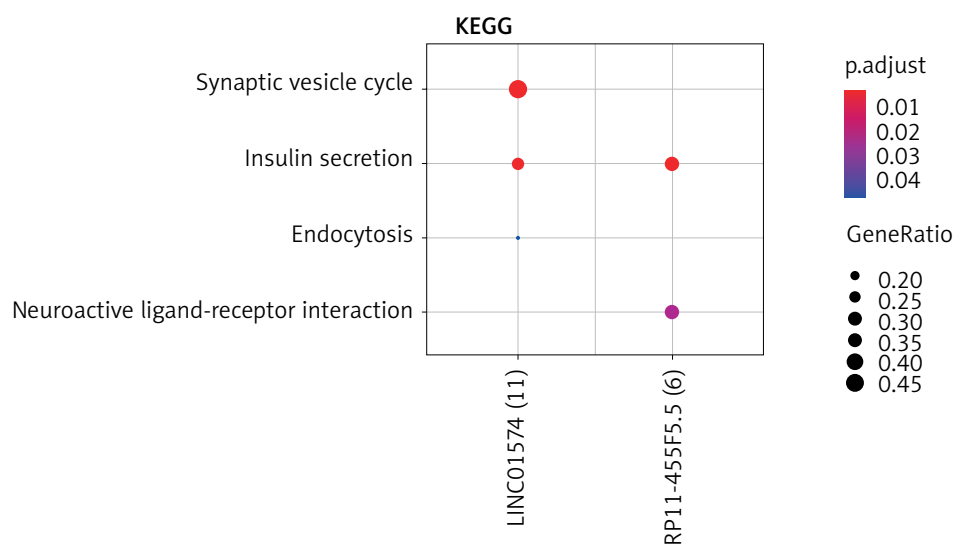

Figure 6. Functional enrichment analysis for IncRNAs in co-expression network. Bubble diagrams show the significantly enriched GO biological processes (A) and KEGG pathways (B) for IncRNAs. Bubble size represents gene count; bubble color from blue to red represents significance from low to high

involved in the development and progression of brain tumor. Extracellular vesicle-mediated crosstalk resulted in elevated synaptic activity in neurons, and inhibiting the release of extracellular vesicles could inhibit glioma growth [31]. Gao et al. demonstrated that SNAP91 expression showed a direct positive correlation with tumor grade and was associated with survival, suggesting that SNAP91 might be a biomarker in GBM [32]. RAB proteins are small GTP-binding proteins that function in intracellular vesicle trafficking. Kim et al. reported that the expression of $R A B 3 A$ was increased in glioma and GBM, and overexpression of $R A B 3 A$ could facilitate proliferation and tumorigenicity of cells [33]. Although LINC01574 has not been investigated in GBM, the results of this study prompted us to speculate that LINC01574 might be implicated in the progression of GBM by regulating the genes involved in the synaptic vesicle cycle.

Other IncNRAs in the co-expression network were found to be enriched in different functions and pathways. RP11-1398P2.1 co-expressed with CDK5R1 was implicated in positive regulation of cyclin-dependent protein kinase activity and cell migration. RP11 has been found to regulate glioma progression [34], tumorigenesis and epithelial-mesenchymal transition [35], and glioma growth [36]. Zhang et al. demonstrated that as a downstream gene of RE1 silencing transcription factor, CDK5R1 was implicated in the proliferation and migration of GBM cells [37]. RP11-455F5.5 co-expressed with MAG (myelin-associated glycoprotein), which exerts a significant effect in inhibiting adhesion and migration of U87MG glioma cells by regulating the Nogo-66 receptor [38]. 
These studies suggested that these IncRNAs had important roles in GBM. However, the expression and functions of these IncRNAs in GBM have not been investigated by experiments, such as real-time quantitative PCR, western blot, and knockdown assays. This is a limitation of this study. Also, the prognostic value of the key IncRNAs has not been confirmed by clinical data.

In conclusion, we identified 18 immune-related prognostic IncRNAs, and established a prognostic risk score model. The risk score model showed the ability to distinguish GBM patients into different risk groups, and patients with high risk scores displayed worse survival. DEGs in high- vs. low-risk groups were involved in the progression of GBM by regulating the synaptic vesicle cycle. This study would provide novel prognostic biomarkers and potential therapeutic targets for the treatment of GBM.

\section{Acknowledgments}

This work was supported by Preferential Subsidy Project to Postdoctoral of Jiangxi Province (grant number 2021KY39) and Innovation Project of Clinical Science and Technology of Jinan City, (grant number 202134057).

\section{Conflict of interest}

The authors declare no conflict of interest.

\section{References}

1. Lah TT, Novak M, Breznik B. Brain malignancies: glioblastoma and brain metastases. Semin Cancer Biol 2020; 60: 262-73.

2. Birzu C, French P, Caccese M, et al. Recurrent glioblastoma: from molecular landscape to new treatment perspectives. Cancers (Basel) 2020; 13: 47.

3. Rajaratnam V, Islam MM, Yang M, Slaby R, Ramirez HM, Mirza SP. Glioblastoma: pathogenesis and current status of chemotherapy and other novel treatments. Cancers (Basel) 2020; 12: 937.

4. Engelhardt B, Vajkoczy P, Weller RO. The movers and shapers in immune privilege of the CNS. Nat Immunol 2017; 18: 123-31.

5. Brown NF, Carter TJ, Ottaviani D, Mulholland P. Harnessing the immune system in glioblastoma. $\mathrm{Br} J$ Cancer 2018; 119: 1171-81.

6. Chen Z, Hambardzumyan D. Immune microenvironmen in glioblastoma subtypes. Front Immunol 2018; 9: 1004.

7. Sasmita AO, Wong YP, Ling APK. Biomarkers and therapeutic advances in glioblastoma multiforme. Asia Pac J Clin Oncol 2018; 14: 40-51.

8. Zhou Y, Yang L, Zhang X, et al. Identification of potential biomarkers in glioblastoma through bioinformatic analysis and evaluating their prognostic value. Biomed Res Int 2019; 2019: 6581576.

9. Han X, Wang X, Li H, Zhang H. Mechanism of microRNA-431-5p-EPB41L1 interaction in glioblastoma multiforme cells. Arch Med Sci 2019; 15: 1555-64.

10. Bhan A, Soleimani M, Mandal SS. Long noncoding RNA and cancer: a new paradigm. Cancer Res 2017; 77: 3965-81.
11. Peng WX, Koirala P, Mo YY. LncRNA-mediated regulation of cell signaling in cancer. Oncogene 2017; 36: 5661-7.

12. Ding Y, Wang X, Pan J, et al. Aberrant expression of long non-coding RNAs (IncRNAs) is involved in brain glioma development. Arch Med Sci 2019; 16: 177-88.

13. Mazor G, Levin L, Picard D, et al. The IncRNA TP73-AS1 is linked to aggressiveness in glioblastoma and promotes temozolomide resistance in glioblastoma cancer stem cells. Cell Death Dis 2019; 10: 246.

14. Lu C, Wei Y, Wang X, et al. DNA-methylation-mediated activating of IncRNA SNHG12 promotes temozolomide resistance in glioblastoma. Mol Cancer 2020; 19: 28.

15. Barrett T, Troup DB, Wilhite SE, et al. NCBI GEO: mining tens of millions of expression profiles - database and tools update. Nucleic Acids Res 2007; 35: D760-5.

16. Bolstad BM, Irizarry RA, Astrand M, Speed TP. A comparison of normalization methods for high density oligonucleotide array data based on variance and bias. Bioinformatics 2003; 19: 185-93.

17. Ritchie ME, Phipson B, Wu D, et al. limma powers differential expression analyses for RNA-sequencing and microarray studies. Nucleic Acids Res 2015; 43: e47.

18. Harrow J, Frankish A, Gonzalez JM, et al. GENCODE: the reference human genome annotation for the ENCODE project. Genome Res 2012; 22: 1760-74.

19. Breuer K, Foroushani AK, Laird MR, et al. InnateDB: systems biology of innate immunity and beyond--recent updates and continuing curation. Nucleic Acids Res 2013; 41: D1228-33.

20. Yu G, Wang LG, Han Y, He QY. clusterProfiler: an R package for comparing biological themes among gene clusters. OMICS 2012; 16: 284-7.

21. Szklarczyk D, Morris JH, Cook H, et al. The STRING database in 2017: quality-controlled protein-protein association networks, made broadly accessible. Nucleic Acids Res 2017; 45: D362-8.

22. Shannon P, Markiel A, Ozier O, et al. Cytoscape: a software environment for integrated models of biomolecular interaction networks. Genome Res 2003; 13: 2498-504.

23. Martikainen M, Essand M. Virus-Based Immunotherapy of Glioblastoma. Cancers 2019; 11: 186.

24. Van Gool SW, Makalowski J, Fiore S, et al. Randomized controlled immunotherapy clinical trials for GBM challenged. Cancers (Basel) 2020; 13: 32.

25. Jackson CM, Choi J, Lim M. Mechanisms of immunotherapy resistance: lessons from glioblastoma. Nat Immunol 2019; 20: 1100-9.

26. Li X, Meng Y. Survival analysis of immune-related IncRNA in low-grade glioma. BMC Cancer 2019; 19: 813.

27. Xia P, Li Q, Wu G, Huang Y. An immune-related IncRNA signature to predict survival in glioma patients. Cell Mol Neurobiol 2021; 41: 365-75.

28. Wang W, Zhao Z, Yang F, et al. An immune-related IncRNA signature for patients with anaplastic gliomas. J Neurooncol 2018; 136: 263-71.

29. Zhou M, Zhang Z, Zhao H, Bao S, Cheng L, Sun J. An immune-related six-IncRNA signature to improve prognosis prediction of glioblastoma multiforme. Mol Neurobiol 2018; 55: 3684-97.

30. Wang X, Gao M, Ye J, et al. An Immune Gene-Related Five-IncRNA Signature for to Predict Glioma Prognosis. Front Genet 2020; 11: 612037.

31. Gao X, Zhang Z, Mashimo T, et al. Gliomas interact with non-glioma brain cells via extracellular vesicles. Cell Rep 2020; 30: 2489-500.e5. 
32. Gao YF, Mao XY, Zhu T, et al. COL3A1 and SNAP91: novel glioblastoma markers with diagnostic and prognostic value. Oncotarget 2016; 7: 70494-503.

33. Kim JK, Lee SY, Park CW, et al. Rab3a promotes brain tumor initiation and progression. Mol Biol Rep 2014; 41: 5903-11.

34. Zhang Y, Mou C, Shang M, Jiang M, Xu C. Long noncoding RNA RP11-626G11.3 promotes the progression of glioma through miR-375-SP1 axis. Mol Carcinog 2020; 59: 492-502.

35. Chang L, Wang J, Zhou F, et al. LncRNA RP11-84E24.3 drives tumorigenesis and epithelial-to-mesenchymal transition of glioma cells by promoting TFAP2C-mediated activation of SNAI1. J Neurooncol 2022; 157: 593.

36. Kang CM, Bai HL, Li XH et al. The binding of IncRNA RP11-732M18.3 with 14-3-3 $\beta / \alpha$ accelerates p21 degradation and promotes glioma growth. EBioMedicine 2019; 45: 58-69.

37. Zhang D, Li Y, Wang R et al. Inhibition of REST suppresses proliferation and migration in glioblastoma cells. Int J Mol Sci 2016; 17: 664.

38. Liao H, Duka T, Teng FY, et al. Nogo-66 and myelin-associated glycoprotein (MAG) inhibit the adhesion and migration of Nogo-66 receptor expressing human glioma cells. J Neurochem 2004; 90: 1156-62. 